\title{
Lead Placement Pseudo Orthogonal XYZ
}

National Cancer Institute

\section{Source}

National Cancer Institute. Lead Placement Pseudo Orthogonal XYZ. NCI Thesaurus. Code C71122.

An electrocardiogram (ECG) lead placement that allows monitoring and recording of cardiac electrical activity. A V1-type lead is used whose positive electrode is localized in the 4 th intercostal space, $2.5 \mathrm{~cm}$ from the sternum. Its negative electrode is placed below the left clavicle. An addition of lead V5 and aVF can be made to facilitate interpretation. 